\title{
SPORADIC VARIATIONS IN WOLF-RAYET STARS
}

\author{
B.S. SHYLAJA \\ Indian Institute of Astrophysics \\ Bangalore 560034 \\ India
}

\begin{abstract}
Many Wolf-Rayet stars display variations which are observable either spectroscopically or photometrically or both. These are not exclusively associated with binary systems. A study is made of the sporadic events in the context of extended atmospheric structure.
\end{abstract}

\section{INTRODUCTION}

Intensive observations of Wolf-Rayet stars in the optical, IR and UV have shown that generally all WR stars are variable. The types of variabilities differ from system to system. For example the variability may be observable in fluxes, radial velocity amplitudes, emission line intensities etc. (For a review, see Moffat, 1990). Multiple periodicities (established in some cases) complicate the analysis.

A detailed investigation of each system reveals that there are sporadic variations in many systems. Such variations might have been observed in any region of the spectrum as an increase in flux or as a change in the line profile. An attempt is made to collect the details of such sporadic variations and understand it in terms of the atmospheric structure.

\subsection{Spectrum Variability}

The well studied binary $\mathrm{CQ} C \mathrm{Cp}$ has interesting sporadic events to be reported. The behaviour of the HeI lines has been changing at some epochs. The line at $\lambda 4471 \AA$ showed line splitting in 1943

(Hiltner, 1943) and in 1952 (Bappu and Viswanadhan, 1977). In 1978 similar line splitting became observable for the HeI $\lambda 3888 \mathrm{~A}$ line (Leung, Moffat and Seggewiss, 1983). In 1950, a sudden change in the strength of HeII line at $\lambda 4686$ was observed (Hiltner, 1950). Spectrophotometric observations show that in 1982 there was probably a similar increase in this line strength (Shylaja, 1986a). It may be noted in all these cases that a corresponding increase in other lines are not seen.

The spectrometric study of HD 50896 (Shylaja, 1986b) showed that only on one occasion there was an increase of flux for HeII line at $\lambda 4860$. Similar spectral variations have been reported a decrease of flux of $N$ IV line at $\lambda 3482$ and an increase in flux for HeII line at $\lambda 4686$ (Van der Hucht et al., 1990).

The spectrophotometric study of HD 76536 (Shylaja, 1990) showed a decrease in flux for carbon lines at $\lambda 4650$, at one epoch only.

K. A. van der Hucht and B. Hidayat (eds.),

Wolf-Rayet Stars and Interrelations with Other Massive Stars in Galaxies, 125-127.

(C) 1991 IAU. Printed in the Netherlands. 
The study on HD 193077 and HD 192103 are reported to show episodic variations (Marshenko, 1988).

\subsection{Light Variability}

A brightening of the order of $0.03 \mathrm{mag}$. was reported for cQ Cep (Hiltner, 1950). Later a variation upto 0.1 mag. was observed in a total duration of 10 months (Kartasheva, 1y/6). Many more systems are being studied for light variability and therefore there is likely to be an increase in such observations.

It may be logical to include here the only one recorded 'eclipse' of WR $113 \equiv \mathrm{CV}$ Ser, since this also may be regarded as a sporadic event in the absence of repeatability. The same argument holds good for WR $22 \equiv$ HD 92740 (Balona et al., 1989) as well. The absence of periodic variations in HD 152270 reported in the same work also may be included as sporadic nature.

\subsection{IR Variability}

Williams et al. (1989) have studied most of the stars of the WC subgroup and found that many are variables. Out of the six variables GL 2179 EW 118 has only one record of variability. Their conclusion is that 'variability in IR from circumstellar dust shells and especially the inner edges is rare', from which they arrive at a constant rate of dust formation.

\section{DISCUSSION}

It is evident from the recorded episodes of sporadic variations, that these variations are preferential to later subgroups in both WN and WC.

Lamontagne and Moffat (1988) have shown that in general the variability is maximum in the $\mathrm{WN} 7$ subgroup. The $\mathrm{WN} 7$ subgroup has also a larger mass loss rate (Doom, 1988).

From a study of the flux variations of CQ Cep (Shylaja, 1986a) it was shown that the eclipse effects are noticeable for only the line of $\mathrm{N} \mathrm{V}$ at $\lambda 4603$. Further, on comparing with similar results of $V 444$ Cyg it was shown that the extent of atmospheres is larger in case of CQ Cep.

This leads us to an important clue that the sporadic variation has its origin in the atmospheric strcuture itself. The stratification prevalent in the atmosphere puts the $\mathrm{N} \mathrm{V}$ and $\mathrm{N}$ IV lines closer to the photosphere and low excitation lines line HeI in the outer regions of the atmosphere. Thus any sporadic phenomena will become immediately apparent in the HeI line structure. This argument is strengthened by looking at the RV curves and flux variations of HeI lines which present a scatter unlike other lines in the same binary system.

The IR sporadic variations can be explained on the basis of nonconstant dust formation rates, although not favoured.

The changes in the mass loss rate associated with a sudden 
change in particle density can explain the sporadic episodes in several cases (for example, HD 152270, Balona et al. 1989 and HD 96548, Gosset and Vreux, 1990). The presence of a companion can lead to such situations when there is a sudden change in mass transfer.

Recently Moffat et al. (1988) have explained small narrow emission bumps moving across an emission profile in terms of rapid blob ejection. Such a theory can be extended to explain the sporadic variations.

Any further work on the interpretation of sporadic events, therefore, needs more data input. An emphasis on the study of HeI lines and a regular monitoring of the systems to 'catch' the event, would facilitate the exclusion of chaotic behaviour as one of the causes.

At the same time it is very important to report such sporadic variations instead of excluding it for purposes of publication.

\section{References}

Balona, L.A., Egan, J. and Marang, F., 1989, MNRAS, 240, 103.

Bappu, M.K.V. and Viswanadham, N., 1977, KOB, A, 2, 89.

Doom, C., 1988, Astron. Astrophys. 192, 170.

Gosset, E. and Vreux, J.M., 1990, Astron. Astrophys. 231, 100.

Hiltner, W.A., 1943, Ap. J., 99, 273.

Hiltner, W.A., 1950, Ap. J., $112,477$.

Kartasheva, T.A., 1976, Sov. Astron. Lett., 2, 197.

Lamontagne, R. and Moffat, A.F.J., 1987, Astron. J. 94, 1008.

Leung, K.C., Moffat, A.F.J. and Seggewiss, W., 1983. Ap. J., 265, 961.

Marshenko, S.V., 1988, Kinematics Phys. Cel. Bodies.

Moffat, A.F.J., 1990, these proceedings.

Moffat, A.F.J., Drissen, L., Lamontagne, R., Roberts, C., 1988, Ap. J., 334, 1038.

Shylaja, B.S., 1986a, J. Astrophys. Astron. 7, 171.

Shylaja, B.S., 1986b, J. Astrophys. Astron, $\overline{7}, 305$.

Shylaja, B.S., 1990, Astrophys. Sp. Sci. 164, 63.

Van der Hucht, K.A., van Genderen, A.M. and Bakker, P.R., 1990, Astron. Astrophys. 228, 108.

Williams, P.M., Van der Hucht, K.A. and The, P.S., 1987, Astron. Astrophys. 182, 91. 


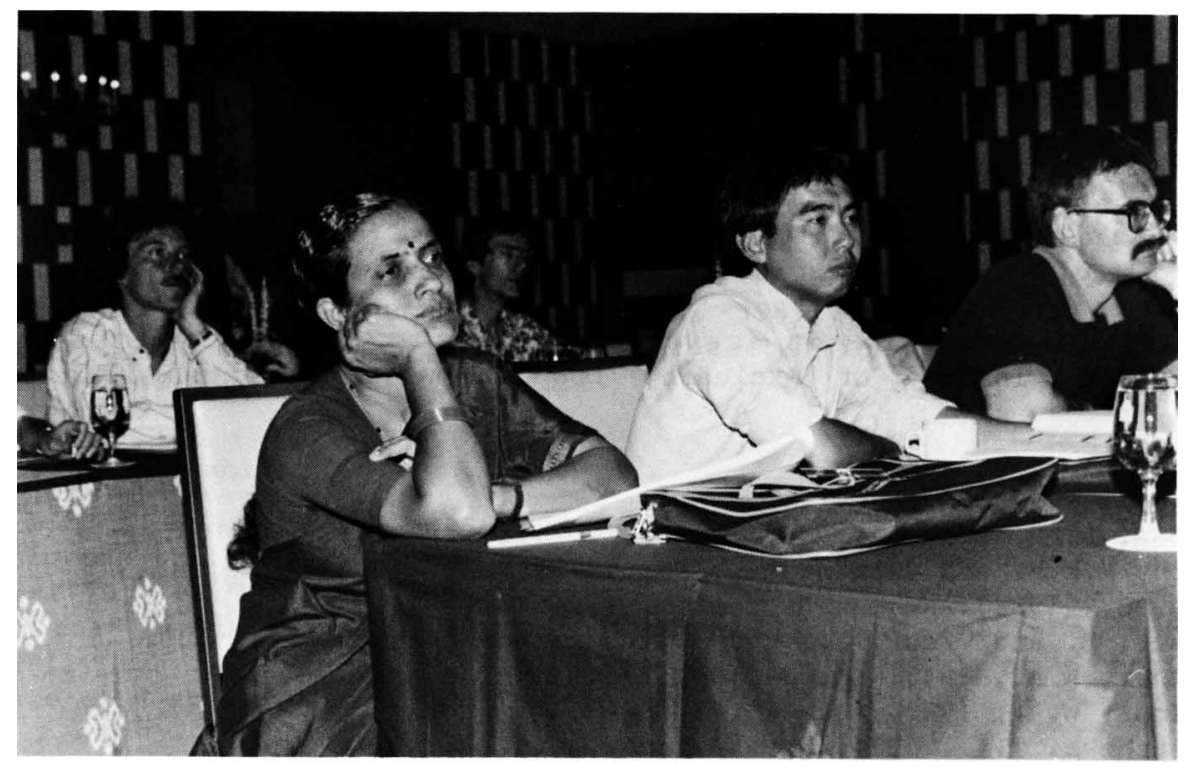

Drissen, Shylaja, Lewis, The, Pollock 